\title{
Extração e Teores de 2-Tridecanona e 2-Undecanona em Genótipos de Tomateiro
}

\author{
Teresinha A. Giustolin ${ }^{1}$, José D. Vendramim ${ }^{1,2}$ e Gilberto C. de Baptista ${ }^{1}$ \\ ${ }^{1}$ Departamento de Entomologia, ESALQ/USP, Caixa postal 9, 13418-900, Piracicaba, SP. \\ ${ }^{2}$ Autor correspondente.
}

\author{
An. Soc. Entomol. Brasil 26(1): 55-60 (1997) \\ Extraction and Contents of 2-Tridecanone and 2-Undecanone \\ in Tomato Genotypes
}

\begin{abstract}
A methodology for extraction of 2-tridecanone (2-T) and 2-undecanone (2-U) from tomato plants (Lycopersicon spp.) was developed using leaves of a commercial cultivar previously fortified with these allelochemicals. Extracts were analyzed in a gas chromatograph, using the following methods: chloroform; chloroform plus Butt extractor; and Butt extractor. The three methods recovered more than $80 \%$ of the allelochemicals. However, the first method was more pratical, using less volume of solvent. The genotypes Lycopersicon hirsutum f. glabratum (PI 134417), L. esculentum ('Santa Cruz Kada AG-373'), and 22 hybrids Fl (RC1) [(PI 134417 x L. esculentum) x PI 134417) were analyzed for the amounts of these allelochemicals. Among the hybrids, six presented both $2-\mathrm{T}$ and $2-\mathrm{U}$, and four presented only $2-\mathrm{T}$. The highest concentration of allelochemicals was $351 \mathrm{ppm}$ of $2-\mathrm{T}$, and $62 \mathrm{ppm}$ of $2-\mathrm{U}$. The allelochemicals were not detected in L. esculentum. The concentrations of 2-T and 2-U in PI 134417 were 1902 and 473 ppm, respectively.
\end{abstract}

KEY WORDS: Insecta, Lycopersicon hirsutum f. glabratum, allelochemicals, plant resistance.

RESUMO - Desenvolveu-se metodologia de extração dos compostos 2-tridecanona (2-T) e 2-undecanona (2-U), em tomateiro (Lycopersicon spp.), utilizando-se folhas de uma cultivar comercial fortificada com os referidos aleloquímicos, sendo os extratos analisados por cromatografia gasosa. Para a extração, testaram-se: clorofórmio; clorofórmio mais extrator de Butt; e extrator de Butt. Os três métodos propiciaram recuperação dos compostos acima de $80 \%$, tendo sido selecionado o primeiro por ser mais prático e utilizar menor volume de solvente. Os genótipos submetidos à extração foram: Lycopersicon hirsutum f. glabratum (PI 134417), L. esculentum ('Santa Cruz Kada AG-373') e 22 híbridos Fl(RC1) [(PI 134417 L. esculentum) x PI 134417]. Dentre os híbridos analisados, em apenas seis foram detectados o 2-T e o 2-U e em outros quatro híbridos apenas o 2-T. No híbrido com maior concentração de aleloquímicos foram encontrados $351 \mathrm{ppm}$ de 2-T e $62 \mathrm{ppm}$ de 2-U. Em L. esculentum, os aleloquímicos não foram detectados, enquanto que na PI 134417, as concentrações de 2-T e 2-U foram, respectivamente, 1902 e $473 \mathrm{ppm}$.

PALAVRAS-CHAVE: Insecta, Lycopersicon hirsutum f. glabratum, aleloquímicos, resistência de plantas. 
O tomateiro selvagem Lycopersicon hirsutum f. glabratum (PI 134417) pode ser utilizado como fonte de resistência a diversos insetos-praga. Williams et al. (1980), procurando determinar as causas da resistência desta espécie, isolaram dos tricomas glandulares de suas folhas o alelo- químico 2-tridecanona (2-T), considerado como fator de resistência. Isto foi confirmado por outros autores como Kennedy et al. (1981), Dimock et al. (1982), Dimock \& Kennedy (1983) e Kennedy (1984). Outro aleloquímico associado aos tricomas glandulares da linhagem PI 134417 é o 2-undecanona (2-U), também aluando adversamente sobre as pragas (Kennedy 1986). Nos programas de melhoramento, visando a utilização destes aleloquímicos como fatores de resistência, é necessário dispor de técnica de extração apropriada. Alguns trabalhos descrevem metodologias de extração destas substâncias (Williams et al. 1980, Kennedy et al. 1981, Dimock \& Kennedy 1983, Lin et al. 1987, Fery \& Kennedy 1987, Weston et al. 1989, Carter et al. 1989), mas nenhum deles comenta sobre a possível perda destas substâncias por evaporação, já que estas são tidas como muito voláteis.

Este trabalho teve como objetivo testar metodologia de extração dos aleloquímicos 2-T e 2-U e quantificações nos seguintes genótipos: L. hirsutum f. glabratum (PI 134417), L. esculentum (Santa Cruz Kada AG-373) e 22 híbridos F 1 (RC1).

\section{Material e Métodos}

Para testar a metodologia de extração dos aleloquímicos 2-tridecanona(2-T) e 2-undecanona(2-U), foi desenvolvido experimento com três métodos três repetições, a saber: clorofórmio; clorofórmio mais extrator de Butt; e extrator de Butt.

No método com clorofórmio utilizaram-se $5 \mathrm{~g}$ de folhas da cv. Santa Cruz Kada AG-373 (L. esculentum), para cada um dos aleloquímicos, que foram fortificadas com $5 \mathrm{mg}$ (1000 ppm) de 2-T e de 2-U, compostos sintéticos com 99\% de pureza (2-T, Fluka Chemical Corp.; 2-U, Pfaltz \& Bauer, Inc.). As folhas contendo os compostos foram submersas em $50 \mathrm{ml}$ de clorofórmio p.a., durante $24 \mathrm{~h}$, em frasco de

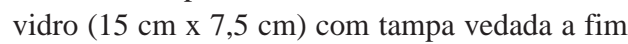
de evitar a evaporação. No método clorofórmio mais extrator de Butt, as $5 \mathrm{~g}$ de folhas fortificadas foram mantidas em cartuchos de papel-filtro por $24 \mathrm{~h} \mathrm{em}$ frasco e o material mantido no extrator de Butt por $4 \mathrm{~h}$. No método com extrator de Butt, após a fortificação e formação dos cartuchos, estes foram colocados diretamente no extrator onde permaneceram por $4 \mathrm{~h}$.

Após a extração, as amostras foram filtradas em papel-filtro, lavadas com $50 \mathrm{ml}$ de benzeno p.a., adicionando-se, $30 \mathrm{~g}$ de sulfato de sódio p.a. granulado, para retirada da água. Os extratos foram filtrados, lavados com $50 \mathrm{ml}$ de benzeno e novamente recolocados nos frascos, onde permaneceram por $24 \mathrm{~h}$. A limpeza dos extratos foi feita em colunas cromatográficas de florisil $(5 \mathrm{~g})$ e eluídas com $30 \mathrm{ml}$ de benzeno. As amostras foram concentradas em evaporador rotativo a vácuo (E. R. V) mantido à $70^{\circ} \mathrm{C}$, até volume entre $4 \mathrm{e} 8 \mathrm{ml}$. Os extratos foram vertidos em tubos de centrífuga graduados e seu volume completado com acetona até $10 \mathrm{ml}$, sendo posteriormente armazenados em "freezer" até o momento das análises.

Para detecção dos aleloquímicos, utilizouse cromatógrafo de gás CG-modelo 3700, equipado com detector de ionização de chama e coluna cromatográfíca de vidro, diâmetro 1/ $8 "$ x 1,8 m fase 2,5\% SE-30, suporte chromosorb W silanizado. As temperaturas de operação foram: 2-T 152,200 e $185^{\circ} \mathrm{C}$;2-U 125,175 e $155^{\circ} \mathrm{C}$, da coluna, detector e vaporizador, respectivamente. Os fluxos dos gases foram respectivamente: 30,210 e $40 \mathrm{ml} / \mathrm{min}$, para o nitrogênio, ar sintético e hidrogênio. O tempo de retenção, tanto para o 2-T como para o 2$\mathrm{U}$, foi de 3'50".

Como as massas injetadas dos padrões e fortificações foram iguais, o cálculo da percentagem de recuperação $(R)$ foi feito através da fórmula: $\mathrm{R}=100 \times \mathrm{hpf} / \mathrm{hpp}$, onde: $\mathrm{hpf}=$ 
altura média do pico de fortificação; e hpp = altura média pico do padrão.

$\mathrm{Na}$ quantificação dos aleloquimicos, os genótipos testados foram oriundos do cruzamento entre a cv. Tropicana-510 (L. esculentum) e a linhagem PI 134417 (L. hirsutum f. glabratum), que deu origem à geração $\mathrm{Fl}$, a qual posteriormente foi retrocruzada com a PI 134417, originando $\mathrm{Fl}(\mathrm{RC} 1)$. Para extração dos aleloquímicos, as folhas foram colhidas de manhã, pesando-se duas amostras (repetições) de $10 \mathrm{~g}$ para cada planta.

Os procedimentos com relação à extração foram semelhantes ao descrito para o método com clorofórmio, exceção do tempo de exposição da amostra ao sulfato de sódio (reduzido para $6 \mathrm{~h}$ ) e dos procedimentos durante a concentração em E.R.V que foi realizada à $50^{\circ} \mathrm{C}$ até que as amostras atingissem volume inferior à $5 \mathrm{ml}$, sendo posteriormente transferidas para vidros de centrífuga graduados, completando até volume de $5 \mathrm{ml}$ com acetona.

As extrações (com duas repetições cada) foram feitas em três etapas, cada uma com um grupo de oito genótipos $\mathrm{Fl}(\mathrm{RC} 1)$, selecionados ao acaso e uma testemunha, constituída da fortificação da cv. com $5 \mathrm{mg}$ (1000 ppm) de 2-T e $5 \mathrm{mg}$ (1000 ppm) de 2-U. Na terceira etapa, incluiu-se também a cv. Santa Cruz Kada AG-373 e a linhagem PI 134417. Assim, o total de análises foi 64 , sendo 12 amostras fortificadas e 52 de campo.

As temperaturas da coluna, do detector e do vaporizador do cromatógrafo nas três extrações dos genótipos analisados foram: $1^{\mathrm{a}}$ e $3^{\mathrm{a}}$ extrações: $146,194,179^{\circ} \mathrm{C}$; $2^{\mathrm{a}}$ extração: 148 , $196,181{ }^{\circ} \mathrm{C}$, respectivamente. Os fluxos de nitrogênio, ar e hidrogênio foram nas três extrações iguais a 30, 210 e $40 \mathrm{ml} / \mathrm{min}$, respectivamente. Os tempos de retenção dos aleloquímicos 2-T e 2-U foram: $1^{\text {a }}$ extração: 3' 50 " e 1'40"; 2a extração: 3'30" e 1'30" e 3 a extração: 3'20" e 1'20", respectivamente.

As médias dos três métodos de extração foram comparadas através do teste " $t$ " de Student ao nível de 5\% de probabilidade.

\section{Resultados e Discussão}

As recuperações de 2-U foram superiores a $90 \%$ nos três métodos de extração, havendo diferença estatística entre os mesmos, sendo o maior valor (107\%) obtido com o extrator de Butt e o menor (91\%) com o clorofórmio mais extrator de Butt (Tabela 1). Resultados semelhantes foram obtidos com 2-T, constatando-se novamente o maior valor de recuperação (106\%) com o extrator Butt e o menor (81\%) com o clorofórmio mais extrator de Butt (Tabela 1). Apesar dos valores de recuperação com o extrator de Butt terem sido superiores aos obtidos com clorofórmio, este método foi mais prático e econômico por utilizar menor quantidade de solvente, e de menor risco, além de permitir percentagem de recuperação de praticamente $100 \%$ para os dois aleloquímicos (Tabela 1).

Tabela 1. Recuperação(\%) dos alelo-químicos 2-undecanona e 2-tridecanona de folhas de tomateiro 'Santa Cruz Kada AG-373' fortificadas com $5 \mathrm{mg}$ de cada composto.

\begin{tabular}{lrr}
\hline Métodos & 2-undecanona' & 2-tridecanona \\
\hline Clorofórmio & $102,0 \pm 5,30 \mathrm{~b}$ & $99,0 \pm 5,90 \mathrm{~b}$ \\
Clorofórmio + Butt & $91,0 \pm 6,52 \mathrm{c}$ & $81,0 \pm 3,51 \mathrm{c}$ \\
Butt & $107,0 \pm 5,20 \mathrm{a}$ & $106,0 \pm 3,51 \mathrm{a}$ \\
\hline
\end{tabular}

${ }^{1}$ Médias seguidas de mesma letra, na coluna, não diferem entre si pelo teste de " $t$ " de Student ao nível de $5 \%$ de probabilidade.

Constatou-se que na primeira extração no tratamento testemunha (fortificação) foram recuperados $88 \%$ do $2-\mathrm{U}$ e $118 \%$ do $2-\mathrm{T}$. Dos oito genótipos analisados nesta etapa, somente nos genótipos 5, 18, 39, 41 e 44 foi detectado o aleloquímico 2-U, registrando-se, em média, uma variação de 3 a 62 ppm (Tabela 2). Em todos os genótipos, com exceção do genótipo 12, detectou-se o 2-T, cujo teor variou, em média, de 15 a $351 \mathrm{ppm}$, valores acima do 
Tabela 2. Concentração dos aleloquímicos 2-undecanona e 2-tridecanona, extraídos com clorofórmio, em genótipos de Lycopersicon spp.

\begin{tabular}{|c|c|c|c|c|}
\hline Extrações & Genótipos & 2-undecanona & 2 -tri & lecanona \\
\hline \multirow{7}{*}{ I } & 5 & $3,0 \pm 1,00$ & 60,0 & 3,09 \\
\hline & 13 & $<\mathrm{LD}^{1}$ & 15,0 & 1,50 \\
\hline & 17 & $<\mathrm{LD}^{1}$ & 50,0 & 3,01 \\
\hline & 18 & $7,0 \pm 1,50$ & 91,0 & $\pm 14,04$ \\
\hline & 39 & $5,0 \pm 4,50$ & 52,0 & $\pm 13,54$ \\
\hline & 41 & $16,0 \pm 3,51$ & 188,0 & $\pm 24,57$ \\
\hline & 44 & $62,0 \pm 13,54$ & 351,0 & $\pm 78,73$ \\
\hline \multirow{3}{*}{ III } & 20 & \multirow{2}{*}{$\begin{array}{l}<\mathrm{LD}^{1} \\
<\mathrm{LD}^{1}\end{array}$} & 8,0 & 0,50 \\
\hline & 35 & & 48,0 & 0,50 \\
\hline & 37 & $4,0 \pm 0,50$ & 30,0 & 0,00 \\
\hline III & PI 134417 & $473,0 \pm 17,55$ & 1902,0 & $\pm 254,26$ \\
\hline
\end{tabular}

${ }^{1} \mathrm{LD}=$ Limite de detecção do método.

limite de detecção (LD) do método (2 ppm) (Tabela 2).

$\mathrm{Na}$ segunda extração, a recuperação do 2U na testemunha (amostras fortificadas) foi de $72 \%$, sendo que somente o genótipo 37 (5 ppm) apresentou este aleloquímico em quantidade acima do limite de detecção. Já com relação ao 2-T, a recuperação na testemunha foi de $95 \%$, sendo este composto detectado apenas nos genótipos 20, 35 e 37, com variação média de 8 a 48 ppm; nos cinco genótipos restantes, não foram detectados nenhum dos dois aleloquímicos (Tabela 2).

$\mathrm{Na}$ terceira extração, as percentagens de recuperação na testemunha foram de $145 \%$ para o 2-U e $109 \%$ para o 2-T. Dos dez genótipos submetidos às análises, nesta etapa, somente na linhagem PI 134417 foram detectados os aleloquímicos (473 ppm de 2-U e 1902 ppm de 2-T) (Tabela 2).

Verificou-se que, em todos os casos em que os compostos foram detectados, houve uma concentração bastante superior de 2-T em relação a 2-U, o que está de acordo com Lin et al. (1987).
Comparando-se as concentrações detectadas na PI $134417(0,19 \%$ de $2-\mathrm{T}$ e $0,047 \%$ de 2-U) com as concentrações destes aleloquímicos $(0,368$ e $0,066 \%$, respectivamente) referidas por Farrar \& Kennedy (1987) para esta mesma linhagem, constatou-se que, para ambos, os valores obtidos foram inferiores. Isto, provavelmente, deveu-se ao fato de que a coleta de folhas foi feita em época de fotofase curta, fator que reduz a produção de 2-T na PI 134417 (Kennedy et al. 1981, Weston et al. 1989).

Considerando-se, por outro lado, os 24 genótipos $\mathrm{Fl}(\mathrm{RC} 1)$ analisados, verificou-se que as concentrações de 2-U e 2-T foram menores, quando comparadas àquelas detectadas na PI 134417, que se caracteriza por apresentar altas concentrações destes aleloquímicos. Assim, observou-se que apenas no genótípo 44 foram detectadas concentrações de 2-T e 2-U superiores a $10 \%$ das respectivas quantidades na linhagem em questão (Tabela 2). A ocorrência de maior proporção de progênies com baixas concentrações de 2-T também foi observada por Fery \& Kennedy (1987) 
e Sorenson et al. (1989) executando um esquema de cruzamento ('Walter' x PI134417) onde obtiveram cerca de $15 \%$ de plantas com baixa concentração de 2-T.

Conclui-se que, apesar de nos diversos genótipos testados (principalmente na PI 134417) terem sido detectadas quantidades inferiores dos dois aleloquímicos quando comparadas às citadas na literatura, a extração feita através da exposição do material vegetal ao clorofórmio, permite análise adequada dos aleloquimicos 2-T e 2-U.

\section{Agradecimentos}

Ao Dr. Wilson R. Maluf (BIOPLANTA) pelo fornecimento de sementes dos genótipos de tomateiro analisados, à Profa. Dra. Marisa A. B. Regitano d'Arce, Departamento de Ciência e Tecnologia Agroindustrial, pelo auxílio e cessão das instalações físicas para a extração dos aleloquímicos, à Dra. Vera L. Ferracini, da EMBRAPA/CNPMA, pelas sugestões apresentadas e à FAPESP pelo auxílio financeiro.

\section{Literatura Citada}

\section{Carter, C. D., J. N. Sacalis \& T.J. Gianfagna.} 1989. Zingiberene and resistance to Colorado potato beetle in Lycopersicon hirsutum f. glabratum. J. Agric. Food Chem. 37: 206-210.

Dimock, M.B. \& G.G. Kennedy. 1983. The role of glandular trichomes in the resistance of Lycopersicon hirsutum f. glabratum to Heliothis zea. Entomol. Exp. Appl. 33: 263-268.

Dimock, M. B., G. G. Kennedy \& W. G. Williams. 1982. Toxicity studies of ana$\log$ of 2-tridecanone, a naturally occurring toxicant from a wild tomato. J. Chem. Ecol. 8: 837-842.
Farrar Jr., R. R. \& G. G. Kennedy. 1987. 2undecanone, a constituent of the glandular trichomes of Lycopersicon hirsutum f. glabratum: Effects on Heliothis zea and Manduca sexta growth and survival. Entomol. Exp. Appl. 43:17-23.

Fery, R.L. \& G.G. Kennedy. 1987. Genetic analysis of 2-tridecanone concentration, leaf trichome characteristics, and tobacco hornworm resistance in tomato. J. Amer. Soc. Hort. Sci. 112: 886-891.

Kennedy, G. G. 1984. 2-tridecanone, tomatoes and Heliothis zea Potential incompatibility of plant antibiosis with insecticidal control. Entomol. Exp. Appl. 35: 305-311.

Kennedy, G. G. 1986. Consequences of modifying biochemically mediated insect resistance in Lycopersicon species, p. 130141. In M.B. Green \& RA. Hedin (eds.), Natural resistance of plants to pests: role of allelochemicals. Amer. Chem. Soc. Symp. Ser. 296.

Kennedy, G. G., R.T.Yamamoto, M. B. Dimock, W. G. Williams \& J. Bordner. 1981. Effect of day length and light intensity on 2-tridecanone leveis and resistance in Lycopersicon hirsutum f. glabratum to Manduca sexta. J. Chem. Ecol. 7: 707-716.

Lin, S.Y. H., J.T. Trumble \& J. Kumamoto. 1987. Activity of volatile compounds in glandular trichomes of Lycopersicon species against two insect herbivores. J. Chem. Ecol. 13: 837-850.

Sorenson, C. E., R. L. Fery \& G. G. Kennedy. 1989. Relationship between Colorado potato beetle (Coleoptera: Chrysomelidae) and tobacco hornworm (Lepidoptera: Sphingidae) resistance in Lycoper- 
sicon hirsutum f. glabratum. J. Econ. Entomol. 82: 1743-1748.

Weston, P.A., D.A. Johnson, H.T. Burton \& J.C. Snyder. 1989. Trichomes secretion composition, trichome densities, and spider mite resistance of ten accessions of Lycopersicon hirsutum. J. Amer. Soc. Hort. Sci. 114: 492-498.
Williams, W. G., G. G. Kennedy, R. T. Yamamoto, J.D. Thacker \& J. Bordner. 1980. 2-tridecanone: A naturally occurring insecticide from the wild tomato Lycopersicon hirsutum f. glabratum. Science 207: 888-889.

Recebido em 07/11/95. Aceito em 15/01/97. 